\title{
Astronomy in Asia
}

\section{Boonrucksar Soonthornthum}

\author{
National Astronomical Research Institute of Thailand (NARIT), Ministry of Science and \\ Technology, Thailand \\ and \\ Department of Physics, Faculty of Science, Chiang Mai University, Chiang Mai 50200, \\ Thailand \\ email: boonrucksar@narit.or.th
}

\begin{abstract}
Astronomy in Asia has continuously developed. Local wisdom in many Asian countries reflects their interest in astronomy since the historical period. However, the astronomical development in each country is different which depends on their cultures, politics and economics. Astronomy in some Asian developing countries such as China and India are well-developed while some other countries especially in south-east Asia, with some supports such as telescopes, training, experts etc. from some developed countries, are trying to promote relevant research in astronomy as well as use astronomy as a tool to promote scientific awareness and understanding for the public. Recently, a new national research institute in astronomy, called the National Astronomical Research Institute of Thailand (NARIT), with a 2.4-metre reflecting telescope has been established in Thailand. One of the major objectives of this research-emphasis institute would aim at a collaborative network among South-East Asian countries so as to be able to contribute new knowledge and research to the astronomical community.
\end{abstract}

Keywords. Asian developing countries, SEAAN, NARIT

\section{Introduction}

It has been aware that the social and economic growth of the countries is dependent on an essential emphasis on education, science and technology. Recently, there has been a great surge in an international effort to foster the development of science and technology in various countries all over the world. International and regional co-operation in basic science research, including astronomy, is promoted in order to ensure high scientific standards. International collaboration has had a very profound impact on research and education in astronomy which, in turn, leads to new discoveries of theories and knowledge that are responsible for our understanding of complex mechanisms of the universe, the behavior of all celestial objects and the evolution of the universe to its present state. Thanks to IAU which strives to promote vital worldwide network and regional co-operation in astronomy to ensure that astronomy becomes a truly shared asset.

As the title suggests, my discussion will be wrapped only on Asia. Asia is one of the world's biggest continents made up of, so to say, extremely developed, least developed, and developing countries. There are significant social and economic differences among these countries. The difference in the scientific and technological infrastructure and in the popularization of science and technology in the two groups of countries are the most important causes of differential social and economical levels. Lack of appreciation of the importance of science as an essential ingredient of economic and social development, and weak educational and scientific infrastructure are the basic problems of the developing countries. The same situation applies to astronomy in Asia, both on basic and advance levels. 
Developed countries have long recognized the necessity and importance of infrastructure and critical size of human resources in the area. In these countries funding scientific enterprise is widely accepted as a vital and long-term investment to a knowledge-based society. The fact is reflected in the amount of investment of the countries the field. Whereas, in developing countries, astronomy has been treated as a 'marginal activity' and perceived as an 'ornament'. Thus, astronomy in developing countries has principal shortcomings in its funding and supporting facilities. Inadequate infrastructure is a critical factor which creates solid barriers to advancement of astronomy in Asian developing countries.

\section{Basic problems encountered in promoting advancement of astronomy in Asian developing countries}

I shall try to summarize some common major factors which have stood up as the obstacles for promoting astronomy in Asian developing countries. The details, of course, are different according to social, cultural, historical, and political elements of each country. They are as follows:

1. Lack of national policy on long-term investment in the field.

2. Lack of planning, management, and support of activities from the government. This, obviously, is a consequence of 1 .

3. Lack of concrete strategies and directions for research work which, in turn, leads to lack of commitment to retain high-level astronomers and researchers to maintain their productivity

4. Lack of vision to encourage collaboration with well-established astronomical institutes or of-like organization in other countries.

5. Lack of infrastructure that is comparable to international standard.

6. Inadequacy to reach the critical size of human resources and the amount of investment in the area.

7. Limited access of relevant information through journals, textbooks, especially internet and electronic communication which now play an integral part of all scientific endeavours.

\section{Strategic plans for development of astronomy in Asian developing countries}

It may be of advantage if we try to pinpoint the strategic plans for development of astronomy in Asian developing countries.

1. Bridging the gap between astronomers and the government.

2. New attitude of astronomers for the development of astronomy in the country.

3. Promote the national awareness and the investment for the development in astronomy in the country.

4. Roles of universities: teaching, research and produce good quality graduates.

5. Providing of the critical mass of researchers and supporting staff in astronomy in the country.

6. The development of standard astronomical infrastructure in the country.

7. Astronomy network in the region mentoring with well-established astronomical institutes. 
Table 1. The number of researchers in south-east Asian developing countries in 2005

\begin{tabular}{ccccc}
\hline Country & $\begin{array}{c}\text { IMF Quota } \\
\text { (millions of } \\
\text { SDR) }\end{array}$ & $\begin{array}{c}\text { Population } \\
\text { (million) }\end{array}$ & Wealth & $\begin{array}{c}\text { Astronomers/ } \\
\text { Population }^{1}\end{array}$ \\
\hline Indonesia & 2079.3 & 222.8 & 9.33 & $0.08(17)$ \\
Malaysia & 1486.6 & 25.4 & 58.52 & $0.28(7)$ \\
Philippines & 879.9 & 83.1 & 10.59 & $0.04(3)$ \\
Thailand & 1081.9 & 64.2 & 16.85 & $0.05(3)$ \\
Singapore & 862.5 & 4.3 & 200.58 & $0.70(3)$ \\
Vietnam & 329.1 & 84.2 & 3.91 & $0.04(3)$ \\
Myanmar & 258.4 & 50.5 & 5.12 & - \\
Laos & 52.9 & 5.9 & 8.97 & - \\
Cambodia & 87.5 & 14.1 & 6.21 & - \\
Brunei & 215.2 & 0.4 & 538.00 & - \\
\hline
\end{tabular}

${ }^{1}$ http//www.iau.org/MEMBERSHIP.10.0.html

\section{New attitudes for developing countries}

Today, in the context of an increasingly globalize trend towards the era of knowledgebased society, developing countries have to embrace a new attitude. Instead of 'asking for donation and support' from developed countries, the concept has gradually switched to the two C's, namely, 'collaboration' and 'co-operation' between countries. Both developed and developing countries have to develop symbiotic relationships to prosper. This means that developing countries have to maneuver the standard of science and technology of their countries to meet the international standard so that collaboration may work out properly. Astronomy is one of the sciences that developed countries have endeavoured to promote and implement in Asian developing countries, focusing on sustained aid to improve the quality and the potential to ensure international standards in research work and academic activities. This has been consolidated in the framework of IAU which is one of the main agencies in the field.

\section{Roles of universities}

The essential prerequisite to any progress is an early recognition of necessity of good education in the area. Therefore the roles of universities in development of astronomy cannot be underestimated. The critical size of human resources and the amount of investment in the area of astronomy illustrates how astronomy is of neglected importance in south-east Asian developing countries. Universities in these countries face shortage of researchers. So, universities need to provide more academic staff and support more training to the existed academic staff. Moreover, universities need to initiate more national and international research collaboration. Table 1 shows number of researchers in south-east Asian developing countries in 2005. Wealth of the country is defined by the ratio of IMF Quota and the population and Astronomers/Population is the number of astronomers, as the individual member of the IAU (in the parenthesis), divided by the population per million of each country, see Hearnshaw (2001). The table shows that the number of astronomers in developing countries is very less and some countries have no astronomer at all. The lack of professional astronomers is one of a critical problems encountered in promoting advancement of astronomy in Asian developing countries. 


\section{Situation in Thailand: the establishment of the National Astronomical Research Institute of Thailand}

Here, I would like to give you some information about the situation in my country. In relevance to this, a new astronomical facility has been established in Thailand recently. On July $20^{\text {th }}$, 2004, the Thai cabinet approved in principle a proposal to establish a National Astronomical Research Institute of Thailand (NARIT) to commemorate King Rama IV's $200^{\text {th }}$ birthday and also to celebrate the $80^{\text {th }}$ birthday of the present King of the Kingdom of Thailand, King Bhumibhol. The main facility of NARIT is the National Observatory which is located on the very top of the highest mountain at the altitude of 2550 metres above the sea level, named Doi Intanon, in Chiang Mai province of northern Thailand, which is also renowned for the superb climate and tourist attractions. The National Observatory will operate a 2.4-metre reflecting telescope with alt-azimuth driving system. This system has been synchronized with automatic motion of the dome structure, so that observation can be carried out efficiently. The National Observatory and the 2.4-metre reflecting telescope are under construction and expect to be operated in the beginning of the year 2009. Figure 1 shows the conceptual design of the National Observatory of Thailand and Figure 2 shows the drawing of the 2.4-metre reflecting telescope which is under construction by EOS Space Systems Pty. Ltd.

Apart from the main observatory and research buildings, there is one more building associated with NARIT, namely, the Information Technology and Training Center for Astronomy (see the conceptual design in Figure 3) which is located at the Office of the Headquarter of Doi Intanon National Park. The centre will provide information and a learning experience in astronomy for the publics, the facilities for remote operations of the National Observatory, a telescope maintenance workshop and accommodation for astronomers.

The office of the Headquarter of NARIT will be located in one of the Chiang Mai University campus which is about $5 \mathrm{~km}$ from the centre of Chiang Mai city. The mission of this research-emphasis institute is to provide the collaborative network for developing and strengthening knowledge in astronomy so as to meet the international standards and will also provide education and learning culture in astronomy for the public and thus encourage them to seek further involvement with science and technology.

\section{The South-East Asian Astronomical Network}

Most of the developing countries in Asia are now aware of the importance of science and technology, including astronomy, and are now attempting to work on sustainable development of basic science. But the awareness does not necessarily make it easy to develop and popularize astronomy, especially in south-east Asian developing countries. As can be seen, the rate of growth and the rate of productivity in promoting astronomy in Asian developing countries vary significantly from region to region. Moreover, south-east Asian developing countries have significantly slower rate than other Asian developing countries.

One major barrier for developing countries in south-east Asia to tackle development issues has been the absence of a defined venue for getting involved. There are few resources outlining the key steps towards cooperation or initiating advancement efforts.

The astronomical network then, is a mindset sorely needed for the development of astronomy challenges facing south-east Asian developing countries and an ever-increasingly globalized world. 


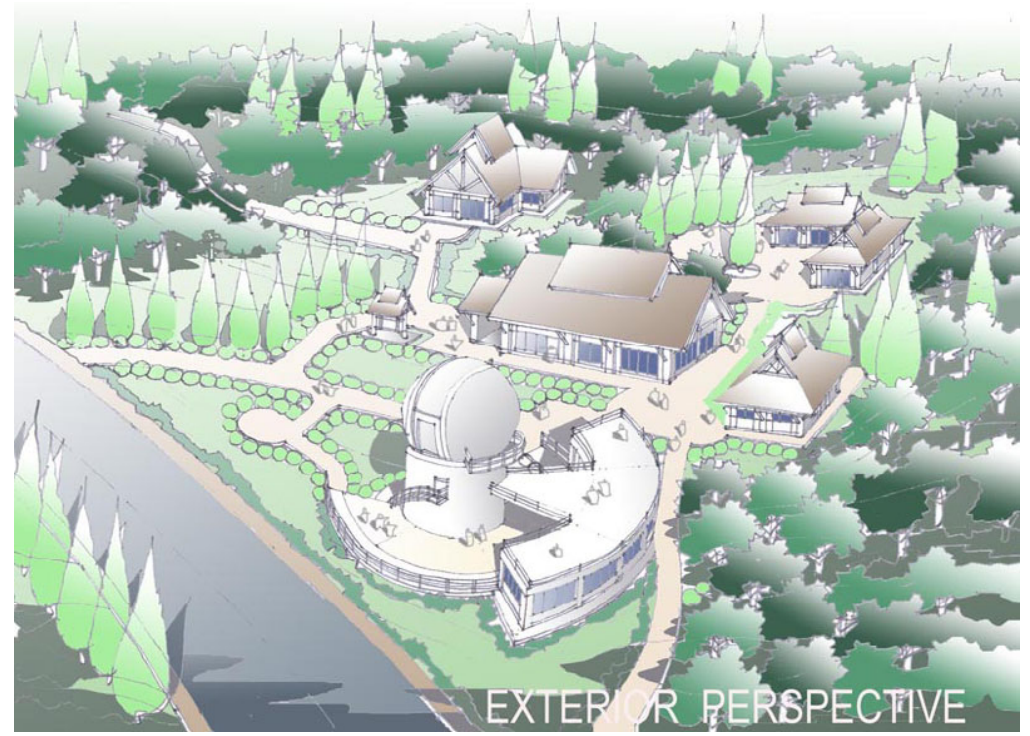

Figure 1. the conceptual design of the National Observatory of Thailand at the summit of Doi Intanon mountain, Chiang Mai, Thailand

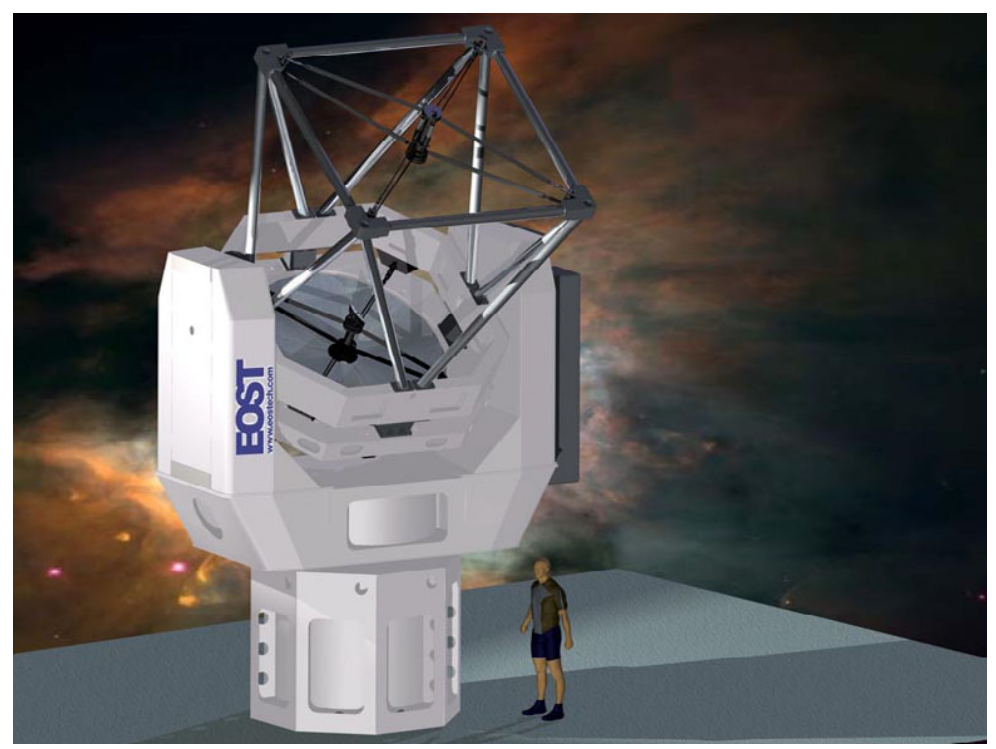

Figure 2. The 2.4-metre reflecting telescope which will be installed at the National Observatory of Thailand

In view of that, I would like to take this opportunity to present the proposal to establish effective mechanisms for nurturing shared development among south-east Asian countries - 'The South-East Asian Astronomical Network (SEAAN)' - with well-established astronomy institutes in some developed countries as mentors. The network will act as a regional platform to create a fruitful advancement in the field of astronomy for member countries. In this way existing resources may be shared and we can work together towards international cooperation. No doubt the IAU will play a central role in pushing this project towards its goal. 


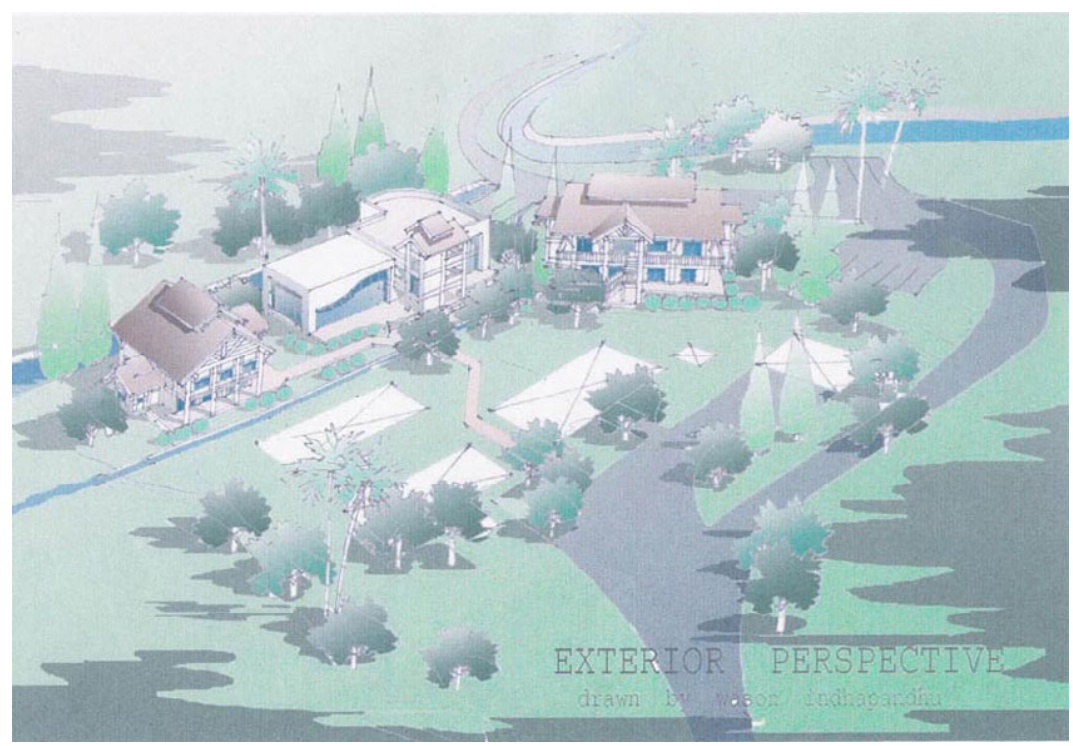

Figure 3. The conceptual design of the Information Technology and Training Center for Astronomy which is located at the Office of the Headquarters of Doi Intanon National Park, Chiang Mai, Thailand

The network, if ever put into action, will make possible for member countries to share information, responsibility in advancement of astronomy in south-east Asia region. Moreover the network will help increasing research capacity and building on achievements.

\section{References}

Hearnshaw, J.B. 2001, in: Alan H. Batten (ed.), Astronomy for Developing Countries, IAU Special Session at the $24^{\text {th }}$ General Assembly, p. 23-27 